\title{
Epidemiology of MdSGHV in Musca domestica
}

\author{
Author: Celeste Vallejo \\ University of Florida \\ cvallejo@ufl.edu
}

\author{
Advisor: Dr. James Keesling \\ University of Florida \\ kees@ufl.edu
}

July 1, 2014

\begin{abstract}
Musca domestica salivary gland hypertrophy virus (MdSGHV) is a disease that enlarges the salivary glands of Musca domestica (the common house fly), as well as causes infertility in female house flies. An infected female fly will no longer produce or lay eggs and will refuse a male's mating attempts. Collaborating with the University of Florida's Entomology and Nematology Department, we have hypothesized that the virus is primarily transmitted through male-male interactions. The constant interaction between males causes damage to the flies' cuticle. When the virus is present on the cuticle, this damage allows the virus entry into the hemocoel, which subsequently infects the internal organs and in particular the salivary glands. The virus adheres to the fly cuticle at contaminated resting sites and contaminated feeding sites. Infection per os is another possible mode of transmission but insufficient to sustain the virus in the fly population. For females, MdSGHV seems to be transmitted largely per os with random occurrences of cuticular damage that take place at the contaminated food and resting sites. There is no evidence that females exhibit aggressive behavior, or that mating causes cuticular damage. Vertical transmission does not seem to be a mode of transmission. We developed a system of differential equations to model the transmission of MdSGHV for a population of Musca domestica at Florida dairy farms. The model is based on the above understanding of the transmission of the virus together with some other simplifying
\end{abstract}


assumptions. A subsequent experiment verified our assumed mode of transmission. This paper illustrates the utility of mathematics in solving problems in other disciplines.

\section{Introduction}

The common house fly, also known as Musca domestica, is an effective vector for a variety of diseases both among humans and amongst house flies themselves. The ability for flies to transmit disease within their own population can be viewed positively because the virus behaves as a biological control agent. Rather than releasing harmful chemicals into the environment to regulate the fly population, flies infected with a disease that is only transmitted amongst themselves can have the same reduction impact without using toxins.

Musca domestica salivary gland hypertrophy virus or MdSGHV is a disease that enlarges the salivary glands in both genders of Musca domestica, as well as causes infertility in female flies. An infected female fly will no longer produce or lay eggs and will refuse a male's mating attempts. Infected male flies become slow to copulate, contrary to normal behavior [3, 6, 8, 9]. Collaborating closely with the University of Florida's Entomology and Nematology Department, a system of differential equations was formulated to model the transmission of MdSGHV through Musca domestica. We hypothesized an additional means by which the virus might be spread. That means was through cuticular damage during male-male interactions. When the virus is on the cuticle, the cuticular damage may allow the virus entry into the hemocoel, causing infection. This hypothesis of cuticular damage as another means of spread of the disease was incorporated in the formation of the differential equations. The results of our simulations mimicked the data gathered at the dairy farms and supported the use of the male interaction parameter within the model.

The model suggested to the entomology collaborators that cuticular damage through male to male interactions could possibly be a valid pathway for the spread of the virus. This led them to devise an experiment in which the damage a fly might experience over the course of its lifetime was simulated by vortexing the flies in a cage. The experiment is explained in more detail in Section 7. The results of the experiment seemed to strongly confirm our conjecture. 
This paper gives a brief introduction to experiments before the formation of our model. These experiments were used as a rationale for the existence of an additional pathway (the interaction parameter) in MdSGHV transmission. Another account of these results that discusses the potential for MdSGHV as a biological control agent appears in the Feature Papers 2013 of the journal Insects [12].

\section{Musca domestica Salivary Gland Hyper- trophy Virus: An Overview}

Discovered in the early 1990s in Florida by Coler et al. [2], MdSGHV is a "non-occluded, enveloped, rod-shaped, double-stranded DNA virus" [6, 7, 9] that is known to exclusively affect adult house flies. It is classified as a hytrosavirus because the salivary glands of the fly are severly impaired, such that the flies become unable to feed, and eventually die. Not only are the salivary glands affected, but also the function and development of the ovaries in female flies $[3,6,9]$. Depending upon the stage in which she becomes infected, the reproductive capacity is either limited or impaired completely. In field settings, females usually mate once for life [10]. Once a female has obtained a sufficient protein meal and oviposition substrates are present, she will lay an egg batch. If the female is infected in the previtellogenic stage (1 to 3 days old) [6], eggs will not even be produced [6, 8]. If she is infected during the postvitellogenic stage (5 to 7 days old) [6] and mates within twenty-four hours of becoming infected, she will lay that single batch of eggs, but no more [6]. After twenty-four hours post-infection, she will refuse male copulation attempts, and will lay no eggs at all [6]. An experiment performed by Lietze et al. [6] demonstrated that when female flies, in both the previtellogenic stage and the postvitellogenic stage, with varying grades of infection were introduced into a healthy male fly population, female flies showed no response to male copulation attempts [6].

Male house flies are similarly affected such that their willingness to copulate with females is suppressed. In a study done by Lietze et al. [6], infected male flies of varying infection stages were introduced into a healthy female fly population. It was found that male flies that had been infected for seventytwo hours were slow to copulate, contrasting to their normal behavior of 
avid copulation that is present even after twenty-four hours post-infection [6]. Suppose there were a house fly population consisting of individuals that have been infected for more than seventy-two hours. Not only would the female flies refuse the males' mating attempts, but the disease would physically impair their ability to produce eggs. Thus, MdSGHV could have the capacity to significanly reduce the Musca domestica population. This makes MdSGHV a possible candidate for use as a biological control agent within a house fly population. A good mathematical model would be helpful in evaluating this possibility. It would require considerable refinement of the model we have produced.

\section{Experiments Before the Introduction of the Model}

It should be noted that there has been no evidence of vertical transmission between house flies and their progeny $[3,6]$. So there must be a way that current generations spread the disease amongst themselves. The most heavily tested hypothesis has been per os transmission $[2,3,6,8,9]$. This has many limitations. For one, the gut of the house fly is lined by a peritrophic matrix, which impedes foreign contaminantes from penetrating the gut's epithelium and entering the hemocoel, the space in which hemolymph circulates, of the fly. Transmission by this means would require that the disease has some manner in which to disrupt the peritrophic matrix. Perhaps in the field there exists microbes or abrasive materials that help to disrupt the peritrophic matrix. This seems unlikely since in an experiment performed by Geden et al. [3], only $1 \%$ of flies were infected when fed cotton strips soaked in a food substrate that had been exposed to infected flies. This low rate of infection may be due to the highly sanitary laboratory conditions in which the flies were held. It has also been hypothesized that there is an immunity component to the virus. In an experiment performed by Lietze et al. [8], when flies less than 24 hours old were force fed the virus, it resulted in an infection range between $30 \%$ and $79 \%$. Then, as flies aged, they became less susceptible to the per os infection route [8]. In the field setting, flies do not begin feeding until after 24 hours $[8,9]$. Therefore, the results are not applicable. The decreased susceptibility and increased resistance to infection as the flies aged implies that there are other routes by which the fly becomes infected; 
feeding on a food source that has been contaminated with virus particles is merely one component.

Another major factor in the transmission of MdSGHV is virus-contaminated environments, such as laboratory cages, that have been exposed to infected flies. In the field, this can be related to contaminated resting sites. Through an experiment performed by Geden et al. [3], uninfected flies that were exposed to cages that had previously held infected flies, but had access to clean food and water, had an infection rate of 3.7\%. Though this does imply that a contaminated environment contributes to the infection rate, the low percentage indicates that this is not the main route in which infection occurs. It is another component that contributes to the overall rate of infection.

Experiments were done in which all possible combinations of infected females and males as well as susceptible females and males were maintained in cages [7]. It was found that the ratio of infected males to susceptible males was higher than the ratio of infected females to susceptible females $[7,8]$, and we believe this is due to the constant physical contact that male flies experience during their lifespan. In an experiment by Lietze et al. [8], virus particles were injected directly into the hemocoel. This resulted in $100 \%$ of the flies becoming infected. This experiment demonstrates that once the virus is introduced into the hemocoel, infection is inevitable. Neither infection per os nor food or environmental contamination (without cuticular damage) have shown to be sufficient to support the rate of infection that is seen in field studies.

Since previous experiments did not produce results similar to field data, we hypothesized the existence of an additional pathway for infection. We hypothesized that interaction between males is a significant culprit for infection for large populations in a small area. We created a model with this additional pathway of infection in which parameters could be adjusted to fit field data. In order to further test this hypothesis, an experiment was performed that simulated interaction through vortexing (Verena-Ulrike Lietze, results unpublished). This experiment seems to confirm conclusively that cuticular damage spreads the virus, regardless of gender. The results were in agreement with the model simulations and collected field data. Section 7 provides more details about the experiment. 


\section{The Model}

The model (1)-(4) is a system of ordinary differential equations: susceptible males $\left(S_{M}\right)$, infected males $\left(I_{M}\right)$, susceptible females $\left(S_{F}\right)$, and infected females $\left(I_{F}\right)$. The two genders were made into separate equations because we believe that the males and the females are infected in different ways. It will be explained further later on in the paper that males are hypothesized to be the perpetuators of the disease within a population of flies. It is a susceptibleinfected model because there is no indication that flies recover from the virus.

The system of differential equations is given below.

$$
\begin{aligned}
\frac{d S_{M}}{d t} & =\delta\left(\left(S_{F}+I_{F}\right)-A \cdot\left(S_{F}+I_{F}\right)^{2}\right)- \\
& -\varepsilon S_{M}-\beta S_{M}-\gamma S_{M}-\alpha \cdot\left(S_{M}+I_{M}\right) \cdot S_{M}-\mu_{1} S_{M} \\
\frac{d I_{M}}{d t} & =\varepsilon S_{M}+\beta S_{M}+\gamma S_{M}+\alpha \cdot\left(S_{M}+I_{M}\right) \cdot S_{M}-\mu_{2} I_{M} \\
\frac{d S_{F}}{d t} & =\delta\left(\left(S_{F}+I_{F}\right)-A \cdot\left(S_{F}+I_{F}\right)^{2}\right)- \\
& -\varepsilon S_{F}-\beta S_{F}-\gamma S_{F}-\mu_{3} S_{F} \\
\frac{d I_{F}}{d t} & =\varepsilon S_{F}+\beta S_{F}+\gamma S_{F}-\mu_{4} I_{F}
\end{aligned}
$$


Table 1: Parameters of the model

\begin{tabular}{crr}
\hline Parameter & \multicolumn{1}{c}{ Description } & Parameter Estimation \\
\hline$\delta$ & Intrinsic growth rate & 0.203152 \\
\hline$\varepsilon$ & Per os transmission rate & $0.0016-(\beta+\gamma)$ \\
\hline$\beta$ & Rate of contamination at the food source & $0.0016-(\varepsilon+\gamma)$ \\
\hline$\gamma$ & Environmental contamination rate & $0.0016-(\varepsilon+\beta)$ \\
\hline$\alpha$ & Rate of infection due to interaction & $0.003 \cdot 10^{-6}$ \\
\hline$A$ & $(\text { Carrying capacity of the total female population })^{-1}$ & $1 \cdot 10^{-6}$ \\
\hline$\lambda_{M}$ & Base proportion of infected males & 0.0076 \\
\hline$\lambda_{F}$ & Proportion of infected females & 0.01 \\
\hline$\mu_{1}$ & Susceptible male daily mortality rate & 0.16 \\
\hline$\mu_{2}$ & Infected male daily mortality rate & 0.21 \\
\hline$\mu_{3}$ & Susceptible female daily mortality rate & 0.08 \\
\hline$\mu_{4}$ & Infected female daily mortality rate & 0.16 \\
\hline
\end{tabular}

\section{Assumptions of the Model}

We assume that the fly population growth is instantaneous and we ignore the time delay in growth as the fly matures from maggot (a stage in which the fly does not become infected) [8,9] to adult. At equilibrium, this does not affect the behavior of the model. The model assumes that the feeding sites and environment have been contaminated at the maximum level and that this level of contamination does not change. It is not known how the virus initially enters the house fly population or the environment. Before equilibrium, the level of contamination at the feeding sites and in the environment will be changing. Taking this change into account would change the dynamics of the model, but it is being ignored. Prior to this model, no method of transmission was found that was sufficient to maintain the infection in the population. This model demonstrates that male aggression and cuticular damage together with environmental contamination and feeding is sufficient to maintain the infection in the population. The Jacobian matrix for (1) - (4) evaluated at the positive equilibrium has eigenvalues with negative real part. Therefore, the model at equilibrium is locally stable.

Experiments that were done previous to our model show that per os infection, as well as contamination at the food source and at the resting sites plays a role in infecting the flies and needs to be included in the model. The 
most notable route of infection is through the interaction term $\alpha$ found only in the susceptible male and infected male differential equations.

One of the differences between our model and a typical infectious disease model is that the virus need only be introduced into the environment for infections to occur. The model does not require an infected individual to be present in the population in order for the suscpetible population to become infected. Once the virus is in the environment, it can adhere to the fly cuticle when the fly comes in contact with it. The presence of the virus on the cuticle makes it possible for the fly to become infected if the cuticle becomes damaged. Damage of this sort occurs in ways that are different for males than for females. Female house flies show only slight damage to their wings as they age $[1,10]$ with likely some damage to the cuticle due to random collisions. In contrast, male house flies exhibit aggressive interactions toward other male flies $[4,7,8,10,11]$. These interactions contribute to the tattered appearance of the males' wings as they age $[1,10]$. We believe that the interaction between males allows for wounding of the cuticle that ultimately allows for the virus, when it is present on the cuticle, to enter the hemocoel. Once the virus is in the hemocoel, the fly inevitably becomes infected [8]. The reader should note that this infection term associated with $\alpha$ is not typical. In a standard susceptible-infected-recovered model the infection spreads to a susceptible only when the susceptible makes contact with an infected. In this model, it is possible for a susceptible male fly to become infected after making aggressive contact with another susceptible male fly through the means described above. A susceptible male may also become infected after interacting with an infected male in the same manner. The fact that the infected fly is infected does not seem to matter. Therefore, the interaction parameter $\alpha$ is represented as a quadratic term in the model and is only in the susceptible male and infected male differential equations. The random cuticular damage for females is represented as a linear term because it occurs in different circumstances, through accidental or random contact.

In the field setting, female flies have a lifespan of approximately two weeks, while males only live for about one week (V.-U. Lietze and C. Geden, personal communication). We assume that infected males and infected females have different mortality rates from their healthy counterparts. Thus, the model assumes: $\mu_{2}>\mu_{1}$ and $\mu_{4}>\mu_{3}$ (V.-U. Lietze and C. Geden, personal communication). Since susceptible males have a higher death rate than susceptible females, infected males will have the highest death rate of the four classifications of flies. This increased death rate is due to the effects 
of the virus. The enlarged salivary glands do not allow for effective feeding: their ability to create saliva, to feed, and to digest their food deteriorates. They become less active and more lethargic before death. Infected female flies do not live as long as susceptible female flies for the same reason. The differences in the mortality rates can be observed in the laboratory, but it is more difficult to determine these in the field. The daily mortality rates are estimated to be: $\mu_{1} \approx 0.16, \mu_{2} \approx 0.21, \mu_{3} \approx 0.08$, and $\mu_{4} \approx 0.16$ (V.-U. Lietze and C. Geden, personal communication), for susceptible males, infected males, susceptible females, and infected females, respectively.

It will be helpful to define $\lambda_{M}\left(\lambda_{F}\right)$ as the ratio of the infected males (infected females) to susceptible males (susceptible females). These ratios will be different due to the interaction parameter $\alpha$. These values are assumed to be much less than one and on the order of $2 \%$ or $1 \%$, respectively (V.-U. Lietze and C. Geden, personal communication).

$$
\begin{gathered}
I_{M}=\lambda_{M} \cdot S_{M} \\
I_{F}=\lambda_{F} \cdot S_{F} \\
0<\lambda_{M}, \lambda_{F} \ll 1
\end{gathered}
$$

To solve for the equilibrium points, we use the system of equations given below.

$$
\begin{aligned}
0 & =\delta\left(\left(S_{F}+I_{F}\right)-A \cdot\left(S_{F}+I_{F}\right)^{2}\right)- \\
& -\varepsilon S_{M}-\beta S_{M}-\gamma S_{M}-\alpha \cdot\left(S_{M}+I_{M}\right) \cdot S_{M}-\mu_{1} S_{M} \\
0 & =\varepsilon S_{M}+\beta S_{M}+\gamma S_{M}+\alpha \cdot\left(S_{M}+I_{M}\right) \cdot S_{M}-\mu_{2} I_{M} \\
0 & =\delta\left(\left(S_{F}+I_{F}\right)-A \cdot\left(S_{F}+I_{F}\right)^{2}\right)- \\
& -\varepsilon S_{F}-\beta S_{F}-\gamma S_{F}-\mu_{3} S_{F} \\
0 & =\varepsilon S_{F}+\beta S_{F}+\gamma S_{F}-\mu_{4} I_{F}
\end{aligned}
$$

From these equilibrium equations we can calculate $S_{M}$ and $S_{F}$, as well as $\lambda_{M}$ and $\lambda_{F}$ using the model at equilibrium (5) - (8), and our assumed 
equations for $I_{M}$ and $I_{F}$.

The values derived from equilibrium are given below.

$$
\begin{aligned}
S_{M}^{*} & =\frac{\mu_{2} \lambda_{M}-(\varepsilon+\beta+\gamma)}{\alpha \cdot\left(1+\lambda_{M}\right)} \\
S_{F}^{*} & =\frac{\delta \cdot\left(1+\lambda_{F}\right)-\left(\varepsilon+\beta+\gamma+\mu_{3}\right)}{\delta \cdot A \cdot\left(1+\lambda_{F}\right)^{2}} \\
\lambda_{M}^{*} & =\frac{\varepsilon+\beta+\gamma+\alpha \cdot S_{M}}{\mu_{2}-\alpha \cdot S_{M}} \\
\lambda_{F}^{*} & =\frac{\varepsilon+\beta+\gamma}{\mu_{4}}
\end{aligned}
$$

We observe that the ratio of infected females to susceptible female flies remains constant. The ratio of infected males to susceptible male flies depends on the susceptible male fly population, and will increase with an increase in the male population. This is due to the interaction parameter and highlights the importance of male-male interactions in the model.

We use the equations obtained from equilibrium (9)-(12) to estimate the remaining parameters, with the exception of $\alpha$. Assuming $1 \%$ of the females are infected $\left(\lambda_{F}\right)$ [3], we can solve for $\varepsilon+\beta+\gamma \approx 0.0016$ using equation (12). Setting $S_{M}=0$ (no susceptible males in the population) and $\varepsilon+\beta+\gamma$ $\approx 0.0016$, we find $\lambda_{M} \approx 0.0076$ as a lower bound for male infection when the infection is at positive equilibrium. We determine $\delta$ in the following manner. We created a Leslie matrix for the female population with a daily survival rate for immatures of $65 \%$ and a daily rate of survival for adults of $95.5 \%$. Ten female eggs are laid by each female each day beginning with the third day after emergence. The largest eigenvalue for this Leslie matrix is 1.22526. We let $\delta$ be the natural logarithm of this dominant eigenvalue. This gives $\delta=0.203152$. We assume that an equal number of male and female eggs are being laid.

\section{Simulations of the Model}

Using the estimated values for the parameters $\delta, \mu_{1}, \mu_{2}, \mu_{3}, \mu_{4}, \varepsilon, \beta, \gamma$ (table 1 ), and fitting $\alpha$ to be $0.003 \cdot 10^{-6}$, we simulated the model for 90 days, a 
typical summer, with $A=\frac{1}{K}$, where $K$ is set to be an arbitrary constant of 1,000,000 (Fig. 1).

Figure 1. A simulation of the differential equation model. The graphs depict the change in population for the Susceptible males; Infected males; Susceptible females; and Infected females. The x-axis is time in days and the $\mathrm{y}$-axis is population size.

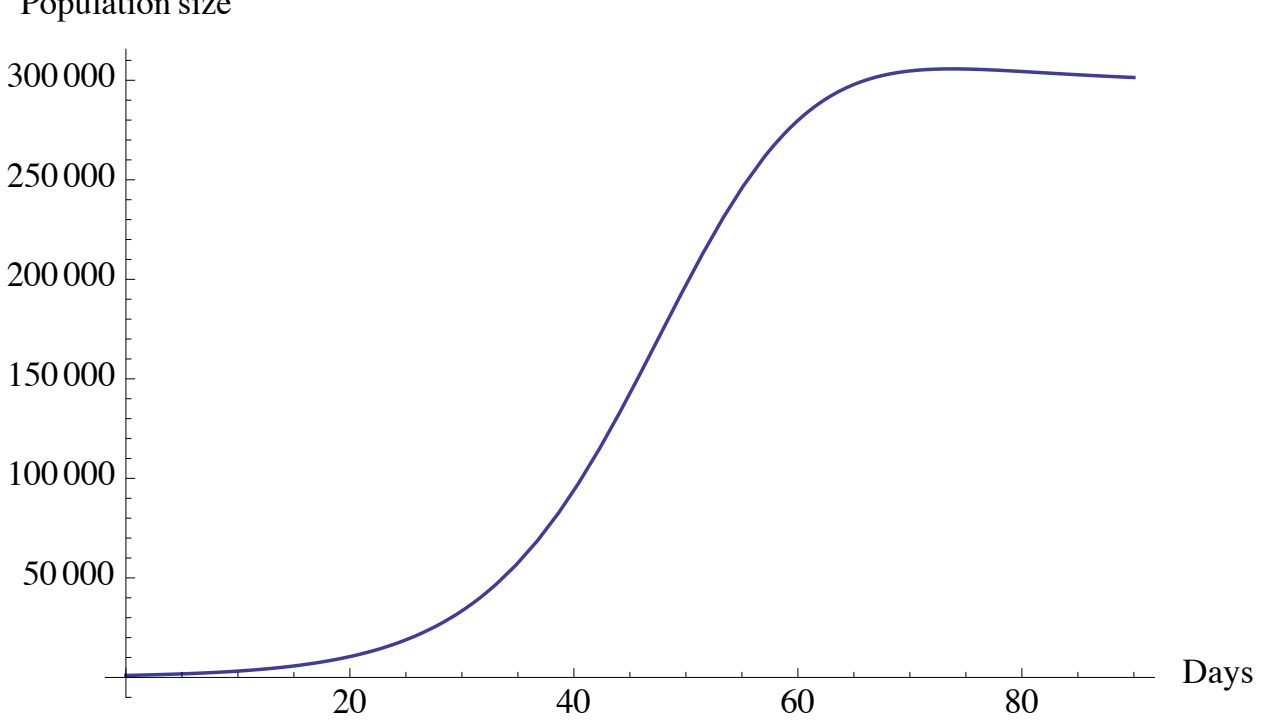




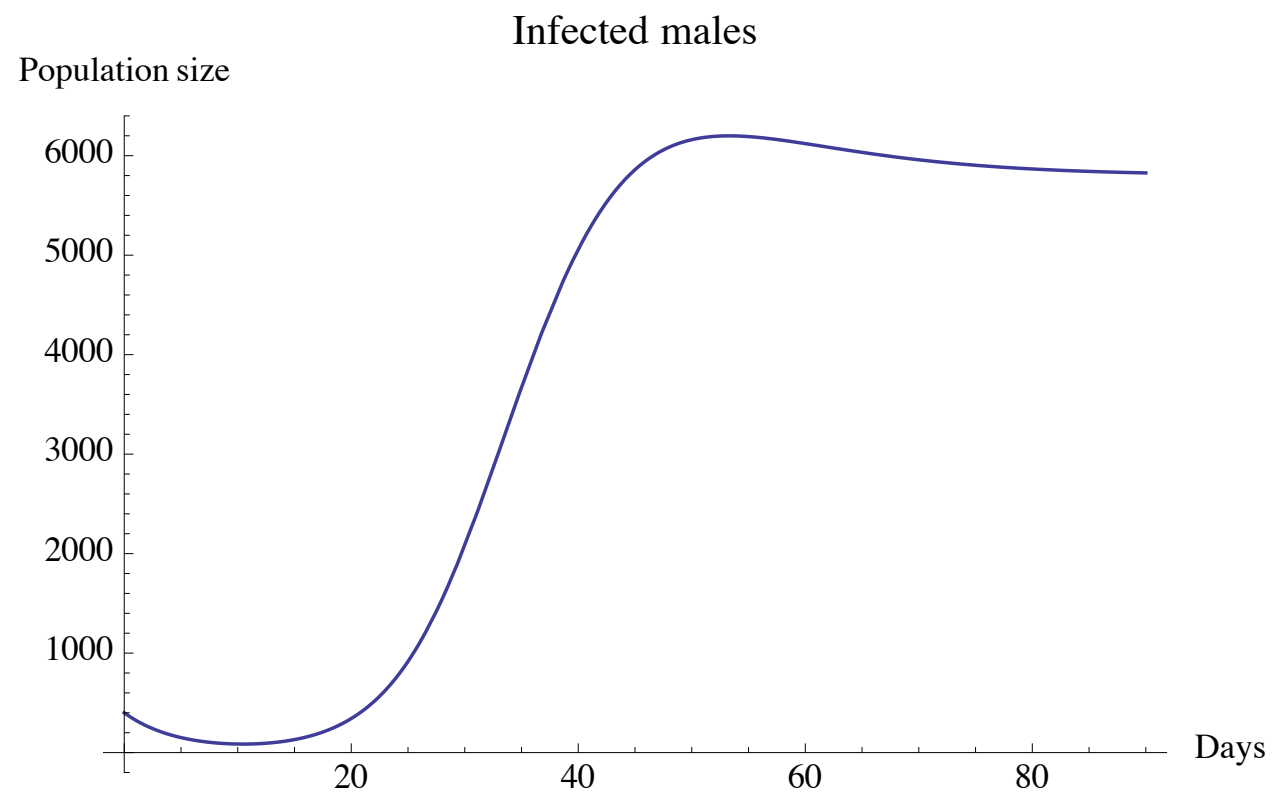

Population size

Susceptible females

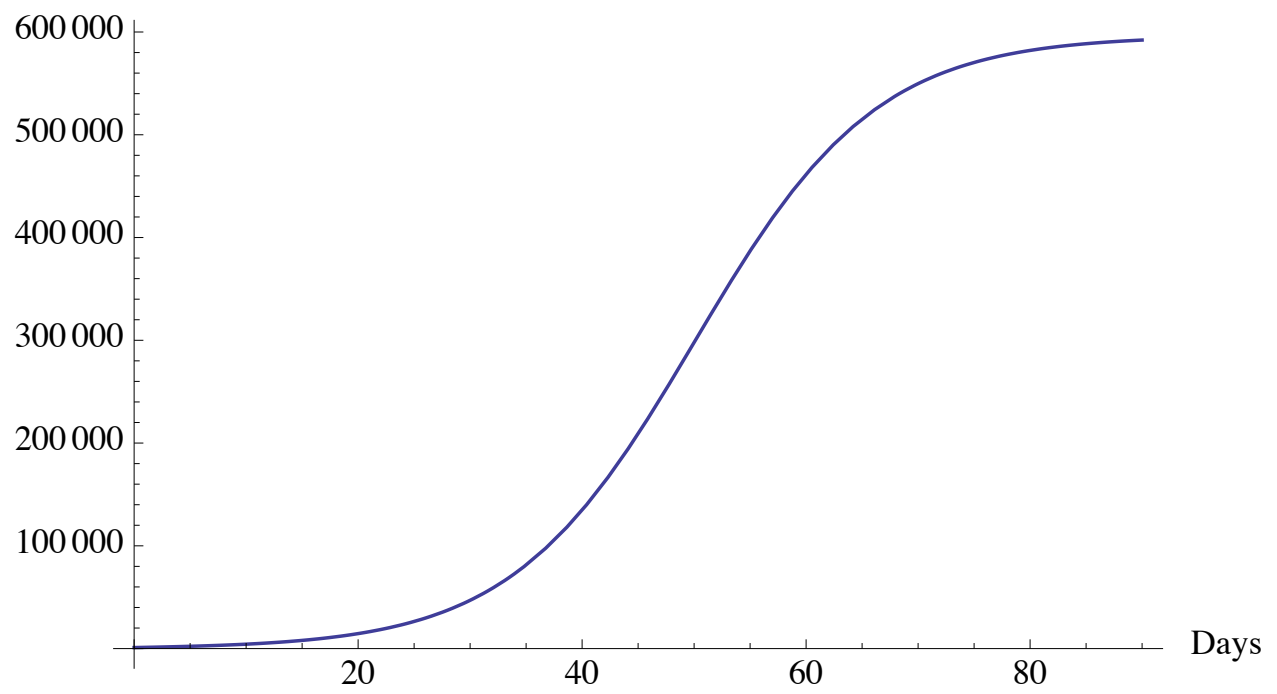


Infected females

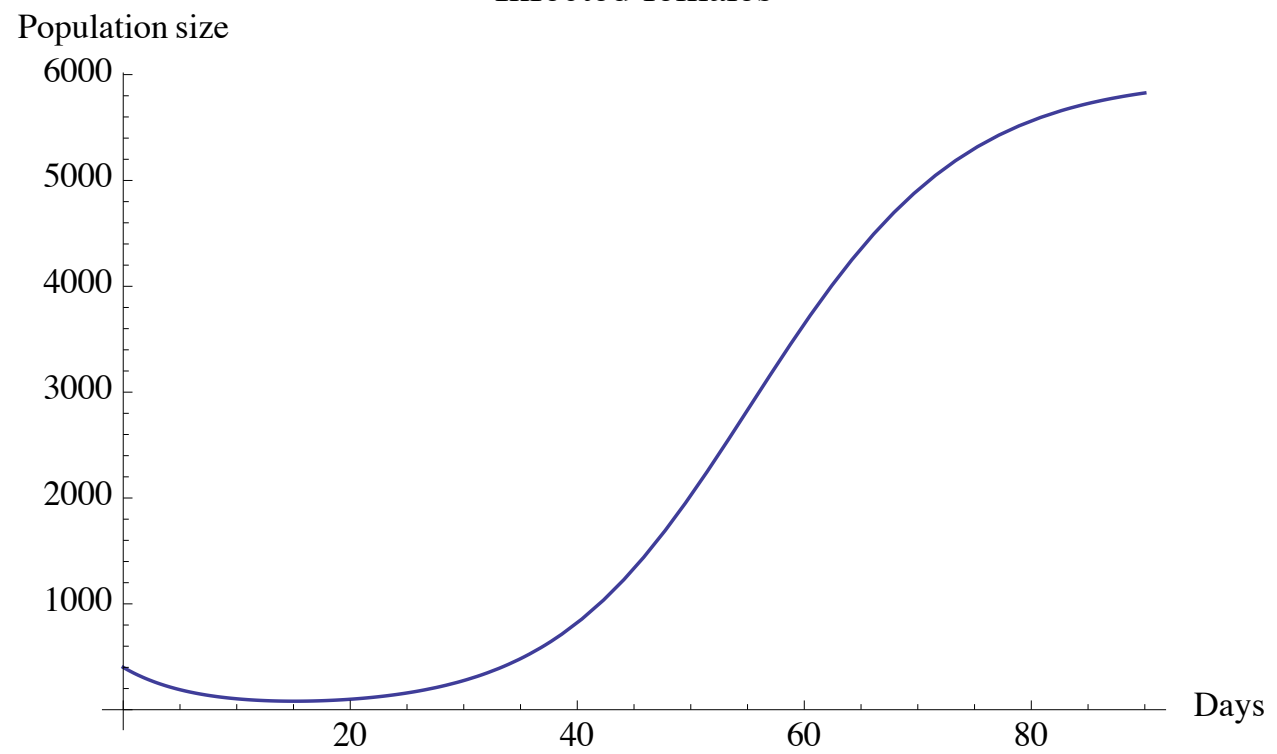


It should be noted that the graph of the infected males reaches equilibrium at two percent of the population of susceptible males and the graph of the infected females is at equilibrium at one percent of the susceptible female population. These results are expected since about $1 \%$ of the susceptible females become infected and about $2 \%$ of the susceptible males become infected in the field (V.-U. Lietze and C. Geden, personal communication).

We observed in C. Geden's laboratory that the flies were aggressive towards each other. Our collaborators indicated that male flies typically exhibit aggressive behavior towards other male flies (V.-U. Lietze and C. Geden, personal communication). We found this to be well-documented in the literature $[4,7,8,10,11]$. The effects of this aggression can be observed through frayed wings and damage to the fly cuticle (V.-U. Lietze and C. Geden, personal communication). This is also documented in the literature $[1,10]$. We hypothesized that the aggressive interactions between male flies that causes cuticular damage is allowing the virus to be introduced into the hemocoel when it is present on the cuticle. This would produce the following cycle. The virus is readily present at the feeding sites through the saliva deposited on the food source as infected flies feed $[3,5,7,8,9,12]$. The virus is also present at resting sites through fecal deposits of infected flies $[3,5]$. It is natural to assume that some of the virus particles present at these sites will adhere to the cuticle of the flies that visit these sites. When the cuticle of a fly is damaged, these virus particles can enter the hemocoel causing infection of the fly, thus completing the cycle. This cycle is how we conjecture that the virus is maintained in the population. In order to test the crucial conjecture in this assumed cycle, an experiment was created to simulate the cuticular damage a fly may receive from aggressive interactions. This experiment is described in Section 7.

\section{The Experiments that Verify Cuticular Dam- age as a Pathway of Infection}

Based on the results of the model, it seemed possible that male interaction causing cuticular damage could be a pathway in the spread of MdSGHV when the virus is present on the fly cuticle. An experiment was devised and performed to see what effect cuticular damage had on the spread of the virus. To simulate the damage a fly may experience as a result of physical contact, 
both male and females flies were vortexed in their cage. The vortexing causes wounding of the cuticle by vibrating the flies against the cage walls and each other. Our suggestion is that the breaks in the cuticle allow the virus entrance to the hemocoel when the virus has adhered to the cuticle. Once the virus is present in the hemocoel, the fly becomes infected [8].

In one experiment, 30 infected flies and 20 healthy flies, in equal gender ratio, were vortexed together in a 72 ounce jar. As a result of the vortexing, $80 \%$ of the healthy males expressed MdSGHV symptoms, while $70 \%$ of the healthy females became infected (Verena-Ulrike Lietze, results unpublished). These results come in stark contrast to the control group that consisted of flies that were not vortexed. In the control group $20 \%$ of the healthy females became infected and $37 \%$ of the healthy males became infected in the same sized cage as those that were vortexed (Verena-Ulrike Lietze, results unpublished). This experiment shows that increased physical contact between flies produces an increased number of infected flies. It should also be noted that the ratio of infected females to susceptible females increased when they were vortexed. This may show that if female flies were to experience as much physical contact as males, then the ratio of infected females to susceptible female flies would increase as well. Since this is not the case, the data supports our hypothesis that the ratio of infected females to suscpetible females is constant and is lower than the ratio of infected males to susceptible males.

Stronger evidence that supports our aforementioned hypothesis is described in the following experiment. Infected flies were placed into a cage for four days. They were removed and replaced with uninfected flies. The uninfected flies were vortexed in this cage. This resulted in $47.7 \%$ of the total population becoming infected (Verena-Ulrike Lietze, results unpublished). The most reasonable explanation for these results is that the vortexing caused damage to the flies' cuticle, allowing MdSGHV entrance into the hemocoel. This caused the infection. These experimental results demonstrate that $\mathrm{cu}-$ ticular damage is a pathway to infection. It also supports the assumption that viral particles are adhering to the cuticle of the flies when they visit contaminated sites. Our model shows that this is a reasonable way in which the virus may be maintained in the fly population. Though the results of the experiment are notable, further experiments need to be performed to fully establish our conjecture. 


\section{Conclusion}

In this paper, we utilize existing knowledge about the transmission of MdS$\mathrm{GHV}$, as well as the previously neglected interaction parameter to develop a system of differential equations to model the transmission of Musca domestica salivary gland hypertrophy virus through a population of house flies. This model includes four pathways of infections, whereas previous research focused on only one route.

Improvements can still be made to the model. The model does not account for the flies that may be infected, but do not yet exhibit the symptoms of MdSGHV. It takes between four and six days for flies to display the symptoms of infection $[3,6,7,9]$. Though PCR is a technique available for early detection of infection, the most cost effective method is through dissecting the fly and visually observing the enlarged salivary glands. Including these flies would create a more complete model. The parameter for per os infection and the parameters for contamination at the resting and feeding sites are grouped together in this model such that $\varepsilon+\beta+\gamma \approx 0.0016$. Differentiating between these pathways and identifying a value for each individual parameter would make the model more accurate.

The constraint $A$ that is used as a limiting factor in the population also needs to be established. There is some factor, whether it be environmental such as availability of food resources or breeding locations, that limits the growth of the population so that it does not extend to infinity. The limiting factor needs to be determined so that the parameter $A$ can be more accurately represented.

To further confirm the model as a viable demonstration of MdSGHV transmission, more experiments need to be completed in the realm of cuticlar damage as a precursor to infection. The vortexing experiment performed by

Lietze (unpublished results) needs to be repeated with a larger sample to accurately simulate the interaction in a field setting.

\section{Acknowledgements}

This work is supported by the University Scholars Program at the University of Florida. The author would like to acknowledge the following individuals in the University of Florida's Entomology and Nematology Department: Dr. Verena-Ulrike Lietze and Dr. Drion Boucias, the individual from the United 
States Department of Agriculture: Dr. Christopher Geden, as well as the individuals from the University of Florida's Mathematics Department: Dr. Jo Ann Lee and Dr. James Keesling for their part in the collaboration. The author would like to express appreciation and thanks to the referee for a thorough review of the paper and very insightful critiques.

\section{References}

[1] G. W. Barber and E. B. Starnes. The activities of house flies. Journal of the New York Entomological Society, 57(4):203-214, 1949.

[2] R. R. Coler, D. G. Boucias, J. H. Frank, J. E. Maruniak, A. GarciaCanedo, and J. C. Pendland. Characterization and description of a virus causing salivary gland hyperplasia in the housefly, Musca domestica. Medical and Veterinary Entomology, 7(3):275-282, 1993.

[3] Christopher Geden, Verena-Ulrike Lietze, and Drion Boucias. Seasonal prevalence and transmission of salivary gland hypertrophy virus of house flies (Diptera: Muscidae). Journal of Medical Entomology, 45(1):42-51, 2008.

[4] E. S. Krafsur, W. C. Black IV, C. J. Church, and D. A. Barnes. Age structure and reproductive biology of a natural house fly (diptera: Muscidae) population. Environmental Entomology, 14(2):159-164, 1985.

[5] Verena-U. Lietze, Kelly R. Sims, Tamer Z. Salem, Christopher J. Geden, and Drion G. Boucias. Transmission of MdSGHV among adult house flies, Musca domestica (diptera: Muscidae), occurs via oral secretions and excreta. Journal of Invertebrate Pathology, 101(1):49-55, 2009.

[6] Verena-Ulrike Lietze, Christopher Geden, Patrick Blackburn, and Drion Boucias. Effects of salivary gland hypertrophy virus on the reproductive behavior of the housefly, Musca domestica. Applied and Environmental Microbiology, 73(21):6811-6818, 2007.

[7] Verena-Ulrike Lietze, Christopher J. Geden, Melissa A. Doyle, and Drion G. Boucias. Disease dynamics and persistence of Musca domestica salivary gland hypertrophy virus infections in laboratory house fly (Musca domestica) populations. Applied and Environmental Microbiology, 78(2):311-317, 2012. 
[8] Verena-Ulrike Lietze, James E. Keesling, Jo Ann Lee, Celeste R. Vallejo, Christopher J. Geden, and Drion G. Boucias. Muscavirus (MdSGHV) disease dynamics in house fly populations - How is this virus transmitted and has it potential as a biological control agent? Journal of Invertebrate Pathology, 112:S40-S43, 2012.

[9] Pannipa Prompiboon, Verena-Ulrike Lietze, John S. S. Denton, Christopher J. Geden, Tove Steenberg, and Drion G. Boucias. Musca domestica salivary gland hypertrophy virus, a globally distributed insect virus that infects and sterilizes female houseflies. Applied and Environmental Microbiology, 76(4):994-998, 2010.

[10] S. S. Ragland and R. S. Sohal. Mating behavior, physical activity and aging in the housefly, Musca domestica. Experimental Gerontology, 8(3):135-145, 1973.

[11] R. S. Sohal and P. B. Buchan. Relationship between physical activity and life span in the adult housefly, Musca domestica. Experimental Gerontology, 16(2):157-162, 1981.

[12] C. R. Vallejo, J. A. Lee, J. E. Keesling, C. J. Geden, V.-U. Lietze, and D. G. Boucias. A mathematic model that describes modes of MdSGHV transmission within house fly populations. Insects, 4(4):683-692, 2013. 